\title{
Acoustic Response of a Sinusoidally Perturbed Hard-Walled Duct
}

\author{
Silvio Del Giudice and Giancarlo Bernasconi \\ Department of Electronics, Information and Bioengineering, Polytechnic University of Milan, Via Ponzio 34/5, 20133 Milan, Italy
}

Correspondence should be addressed to Silvio Del Giudice; delgiudice@elet.polimi.it

Received 2 August 2013; Revised 18 October 2013; Accepted 19 October 2013

Academic Editor: Carlo Cattani

Copyright ( 2013 S. Del Giudice and G. Bernasconi. This is an open access article distributed under the Creative Commons Attribution License, which permits unrestricted use, distribution, and reproduction in any medium, provided the original work is properly cited.

\begin{abstract}
Acoustic wave propagation in hard-walled ducts is of interest in many fields including vehicle design, musical instruments acoustics, and architectural and environmental noise-control. For the case of small sinusoidal perturbation of the cross-section, it is possible to derive simple though approximate analytical formulas of its plane wave acoustic reflection and transmission spectral response that resembles the optical situation of uniform Bragg gratings. The proof is given here, starting from the "horn equation" and then exploiting the coupled-modes theory. Examples of the results obtained with these analytical formulas are shown for some sinusoidally perturbed ducts and compared to results obtained through a numerical method, revealing a very good agreement.
\end{abstract}

\section{Introduction}

The propagation of waves in periodic media has received much attention in the past in different fields of physics: a comprehensive review can be found in Elachi [1], with references on the propagation of acoustic waves in ducts with sinusoidally perturbed walls [2-4]. An interesting feature of wave propagation in periodic media discussed in the review is the existence of stopbands and passbands related to the medium periodicities.

In the last decades much research effort was dedicated to the theoretical and experimental study of elastic wave propagation in periodic waveguides. For instance, Fokkema [5] dealt with periodic boundaries of elastic media; other authors studied waves propagating along periodically corrugated plates [6-10] and along ducts [11-15].

The purpose of this paper is not to advance the research work accomplished so far, but rather to provide an approximate simplification of the established theory, when proper hypotheses are satisfied. Attention is indeed limited to acoustic propagation in hard-walled ducts whose cross-section undergoes a small sinusoidal perturbation with respect to a reference mean value. The work holds for any filling fluid, typically air, provided that the hard-wall hypothesis is verified.
Like in Munday et al. [15], the analysis is restricted to onedimensional (plane wave) propagation, where the waveguide geometry is defined simply by the cross-section along the axial coordinate. The starting point of the theoretical analysis is the Webster horn equation, as performed by Nagarkar and Finch [16], who studied sinusoidal horns, and by Griffiths and Steinke [17], who reviewed the theory of one-dimensional wave propagation in locally periodic media consisting of an arbitrary number of identical cells and showed the acoustic solution for some particular geometries. Lau and Campos [18] also solved the acoustic wave equation for onedimensional propagation along a duct with a small wall sinusoidal perturbation: the exact solutions were obtained as power series expansions around the middle of the duct.

Recently, Hawwa [19] analyzed sound waves in a circular cylindrical duct having a geometric periodicity at its wall, by solving numerically the wave equations.

In optics, or more general in electromagnetics, a periodically perturbed medium is called a Bragg grating, or simply a multilayer medium: the transmission and reflection of a uniform grating can be expressed with simple closed-form formulas (Kogelnik [20]). The acoustic analog of the uniform Bragg grating is a duct whose cross-section sinusoidally varies but, to the authors' knowledge, a simple formula for 


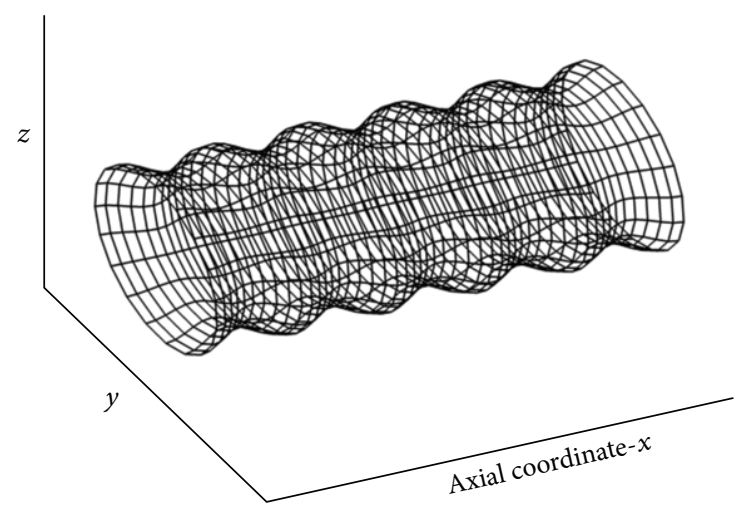

FIGURE 1: Duct with a periodic perturbation of the cross-section.

the acoustic response of such a waveguide is not available in literature.

This paper exploits thus the Bragg gratings theory to solve the acoustic "horn equation," obtaining simple formulas for the reflection and transmission spectral response of the waveguide as a function of acoustic and geometric parameters, under the hypothesis of a small sinusoidal cross-section variation compared to the mean reference value.

Simple and closed-form solutions are advantageous in modeling/inversion procedures, as, for example, in bore reconstruction, design of noise-control devices (employed, e.g., for jet engines or HVAC systems), and even monitoring of transportation pipelines.

The following sections describe the scenario, the mathematical derivation, and the result for some example cases.

The solution provided can take into account also wave attenuation, typically hard-wall losses (boundary layer friction), whose terms are added a posteriori.

\section{Theory}

The scenario considered here is a circular hard-walled duct with a small periodic perturbation of the cross-section along the axial coordinate (Figure 1). Two similar though analytically different geometric cases are considered: one is when a sinusoidal function describes the variation of the cross-section; the other when a sinusoidal function describes the variation of the radius of the circular cross-section.

As in Lau and Campos [18], the acoustic wavelength is supposed to be larger than the transverse dimensions of the duct, so that only the fundamental longitudinal mode exists. Moreover, the changes in cross-section are supposed not to be too rapid with respect to the transverse dimension, so that the wavefronts remain approximately plane.

Under these hypotheses the governing geometrical parameters reduce to the cross-section area (the shape can be neglected), and the starting physical law is the onedimensional "horn equation" [17]:

$$
\frac{\partial^{2} \Psi}{\partial t^{2}}=v^{2}\left(\frac{\partial^{2} \Psi}{\partial x^{2}}+\frac{1}{S} \frac{d S}{d x} \frac{d \Psi}{d x}\right)
$$

The field variable $\Psi$ is the pressure over ambient, $S$ is the cross-section area, $v$ is the phase velocity, $x$ is the waveguide axial coordinate, and $t$ is the time.

Let us consider harmonic waves:

$$
\Psi=\psi(x) e^{-i \omega t}
$$

The substitution of (2) in the wave equation (1) yields

$$
\frac{\partial^{2} \psi}{\partial x^{2}}+\frac{1}{S} \frac{d S}{d x} \frac{d \psi}{d x}+\beta^{2} \psi=0
$$

where $\beta=\omega / v$ is the acoustic wavenumber.

From this point, the mathematical steps and the approximations introduced follow the computation of the transfer function for a uniform optical fiber Bragg grating (Erdogan [21] and Kogelnik [20]), with adaptation to the acoustic waveguide case.

The pressure field is expressed as a linear combination of the fundamental modes propagating in the opposite directions

$$
\psi(x)=A(x) e^{-i \beta x}+B(x) e^{i \beta x},
$$

where, according to the adopted time convention, $A$ and $B$ are the amplitudes of the waves propagating in the $-x$ and $+x$ directions, respectively.

The first derivative of (4) is

$$
\psi^{\prime}=A^{\prime} e^{-i \beta x}-i \beta A e^{-i \beta x}+B^{\prime} e^{i \beta x}+i \beta B e^{i \beta x},
$$

with prime denoting derivative with respect to $x$.

In the hypothesis of weak coupling between the two modes, the second derivatives of $A$ and $B$ are neglected, because $A^{\prime \prime} \ll \beta^{2} A$ and $B^{\prime \prime} \ll \beta^{2} B$; therefore, the second derivative of (4) becomes

$$
\psi^{\prime \prime} \cong-2 i \beta A^{\prime} e^{-i \beta x}-\beta^{2} A e^{-i \beta x}+2 i \beta B^{\prime} e^{i \beta x}-\beta^{2} B e^{i \beta x} .
$$

Substituting the expressions for derivatives, and multiplying by $e^{i \beta x}$, the wave equation (3) yields

$$
\frac{S^{\prime}}{S}\left(A^{\prime}-i \beta A+B^{\prime} e^{2 i \beta x}+i \beta B e^{2 i \beta x}\right)-2 i \beta A^{\prime}+2 i \beta B^{\prime} e^{2 i \beta x}=0 \text {. }
$$

The solution of (7) requires a system of two differential equations for $A$ and $B$. Therefore $A^{\prime}$ and $B^{\prime}$ are alternately isolated from (7), neglecting in each resulting equation the dependency on the derivative of the other coefficient. This is justified by the fact that $S$ and so $A$ and $B$ are slowly variable functions of $x$ [21].

The resulting system is

$$
\begin{aligned}
& A^{\prime}=\frac{i \beta S^{\prime} / S}{S^{\prime} / S-2 i \beta} A+\frac{-i \beta S^{\prime} / S}{S^{\prime} / S-2 i \beta} B e^{2 i \beta x}, \\
& B^{\prime}=\frac{-i \beta S^{\prime} / S}{S^{\prime} / S+2 i \beta} B+\frac{i \beta S^{\prime} / S}{S^{\prime} / S+2 i \beta} A e^{-2 i \beta x},
\end{aligned}
$$


that can be expressed in this way:

$$
\begin{aligned}
& A^{\prime}=-i c_{11} A-i c_{12} B e^{2 i \beta x}, \\
& B^{\prime}=-i c_{22} B-i c_{21} A e^{-2 i \beta x} .
\end{aligned}
$$

Under the hypothesis of slow section perturbations, and far from the null frequency, it is $\left|S^{\prime} / S\right| \ll|2 i \beta|$, and the coefficients $c_{i j}$ in (9) become

$$
\begin{aligned}
& c_{11}=-\frac{\beta S^{\prime} / S}{S^{\prime} / S-2 i \beta} \cong \frac{S^{\prime}}{2 i S}, \\
& c_{12}=\frac{\beta S^{\prime} / S}{S^{\prime} / S-2 i \beta} \cong-\frac{S^{\prime}}{2 i S}, \\
& c_{21}=-\frac{\beta S^{\prime} / S}{S^{\prime} / S+2 i \beta} \cong-\frac{S^{\prime}}{2 i S}, \\
& c_{22}=\frac{\beta S^{\prime} / S}{S^{\prime} / S+2 i \beta} \cong \frac{S^{\prime}}{2 i S} .
\end{aligned}
$$

Now, the two aforementioned geometric cases are considered; first, when the cross-section is a cosine function of $x$; that is,

$$
S(x)=s \cos (\gamma x)+s_{0},
$$

and second, when the radius of the circular cross-section is a cosine function of $x$; that is,

$$
S(x)=\pi\left(s \cos (\gamma x)+s_{0}\right)^{2} .
$$

$\gamma$ is the perturbation wavenumber, $s$ is the perturbation amplitude, and $s_{0}$ is the mean value.

The ratio between the derivative of the cross-section and the cross-section itself $S^{\prime} / S$, for $s \ll s_{0}$, is approximately

$$
\frac{S^{\prime}(x)}{S(x)} \cong-4 \kappa \sin (\gamma x)=2 i \kappa\left(e^{+i \gamma x}-e^{-i \gamma x}\right),
$$

where $\kappa=s \gamma / 4 s_{0}$ for the cross-section sinusoidal perturbation and $\kappa=s \gamma / 2 s_{0}$ for the radius sinusoidal perturbation.

The system (9) becomes

$$
\begin{aligned}
& A^{\prime}=-i \kappa\left(e^{+i \gamma x}-e^{-i \gamma x}\right) A+i \kappa\left(e^{+i \gamma x}-e^{-i \gamma x}\right) B e^{2 i \beta x}, \\
& B^{\prime}=-i \kappa\left(e^{+i \gamma x}-e^{-i \gamma x}\right) B+i \kappa\left(e^{+i \gamma x}-e^{-i \gamma x}\right) A e^{-2 i \beta x} .
\end{aligned}
$$

The terms that contain a rapidly oscillating dependence with $x$ can be neglected: they correspond to the complex exponentials with high phase constant (in magnitude) compared to the others, that is, $e^{ \pm i \gamma x}, e^{2 i \beta x+i \gamma x}$, and $e^{-2 i \beta x-i \gamma x}$ [21].

The result is

$$
\begin{aligned}
& A^{\prime}=-i \kappa B e^{i(2 \beta-\gamma) x}, \\
& B^{\prime}=i \kappa A e^{-i(2 \beta-\gamma) x} .
\end{aligned}
$$

By performing in the system (15) the following substitutions:

$$
\begin{aligned}
& a=A e^{-i(\beta-\gamma / 2) x}, \\
& b=B e^{i(\beta-\gamma / 2) x},
\end{aligned}
$$

and calling $\sigma=\beta-\gamma / 2$, one obtains

$$
\begin{aligned}
& a^{\prime}=-i \sigma a-i \kappa b \\
& b^{\prime}=i \sigma b+i \kappa a .
\end{aligned}
$$

The system (17) is a standard system of two coupled first-order ordinary differential equations with constant coefficients, for which closed-form solutions can be found, when appropriate boundary conditions are specified.

Since $A$ corresponds to the backward propagating wave and $B$ to the forward propagating one, the boundary conditions, for a forward propagating wave impinging the waveguide at $x=0$, are

$$
\begin{aligned}
& A(L)=0 \\
& B(0)=1
\end{aligned} \Longrightarrow \begin{aligned}
& a(L)=0 \\
& b(0)=1,
\end{aligned}
$$

where $L$ is the length of the sinusoidally perturbed duct.

The solution of system (17) with boundary conditions (18) is obtained by means of linear algebra:

$$
\begin{aligned}
& a(x)=\frac{i \kappa\left(e^{-\delta L} e^{\delta x}-e^{\delta L} e^{-\delta x}\right)}{e^{\delta L}(i \sigma-\delta)-e^{-\delta L}(i \sigma+\delta)}, \\
& b(x)=\frac{e^{\delta L} e^{-\delta x}(i \sigma-\delta)-e^{-\delta L} e^{\delta x}(i \sigma+\delta)}{e^{\delta L}(i \sigma-\delta)-e^{-\delta L}(i \sigma+\delta)} .
\end{aligned}
$$

The reflection and transmission spectral responses $R(\omega)$ and $T(\omega)$ correspond, respectively, to $a(0)$ and $b(L)$. Hence, finally

$$
\begin{aligned}
& R(\omega)=\frac{i S \sinh \delta L}{\cosh \delta L-i P \sinh \delta L}, \\
& T(\omega)=\frac{1}{\cosh \delta L-i P \sinh \delta L},
\end{aligned}
$$

where

$$
\begin{aligned}
& \delta=\sqrt{\kappa^{2}-\sigma^{2}}, \\
& S=\frac{\kappa}{\delta}, \\
& P=\frac{\sigma}{\delta} .
\end{aligned}
$$

According to their definition, the spectral responses $R$ and $T$ refer to the acoustic pressure amplitude: the corresponding power spectral responses can be found by taking the square of their magnitude. In absence of attenuation, the sum of the power spectral responses is unitary, as expected.

Equations (20) are the acoustic analog of the optical formulas for the uniform Bragg grating in Kogelnik [20], and the parameters $\kappa$ and $\sigma$ are expressed here as a function of acoustic wave parameters and waveguide geometric parameters.

The reflection spectral response has a maximum for $\sigma=$ 0 , corresponding to the frequency $f_{\max }=\gamma v / 4 \pi$. For the same frequency the transmission response has a minimum.

Finally, even if the considered one-dimensional wave equation does not contain a loss term, one may add the 


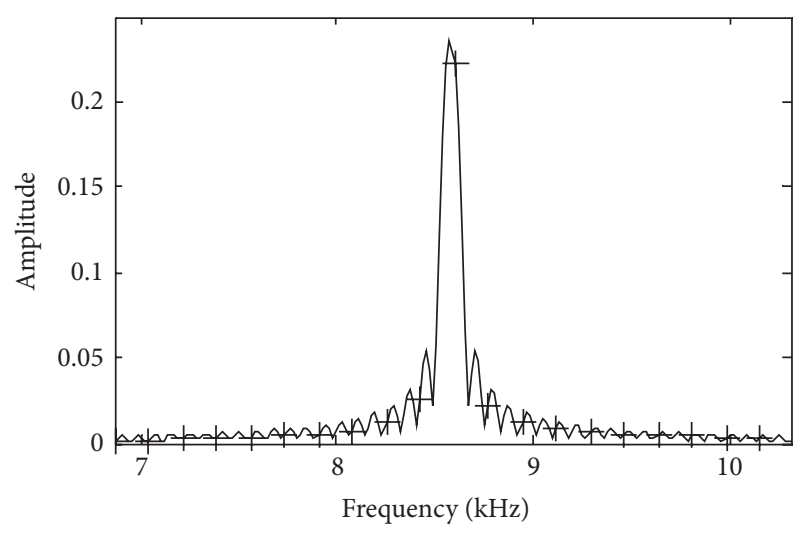

(a)

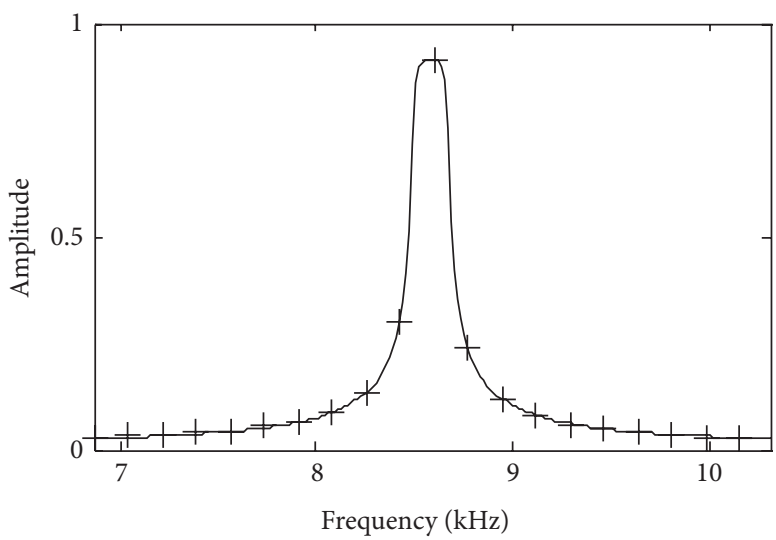

(c)

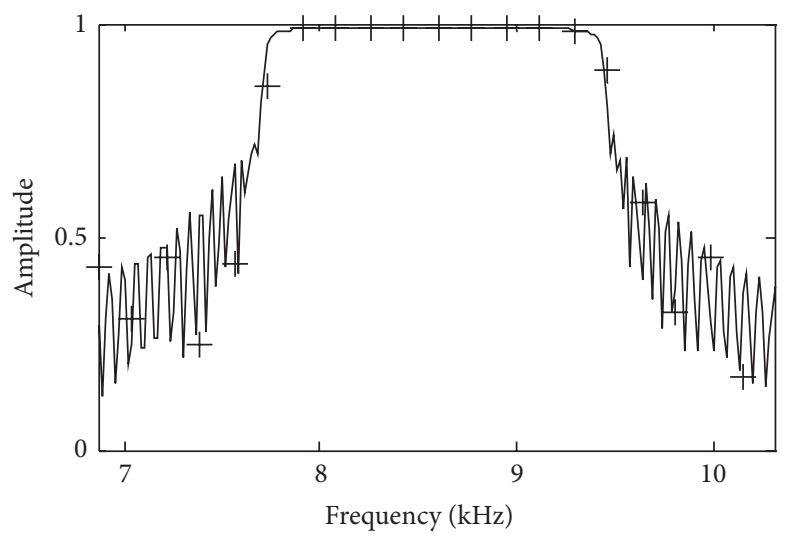

(e)

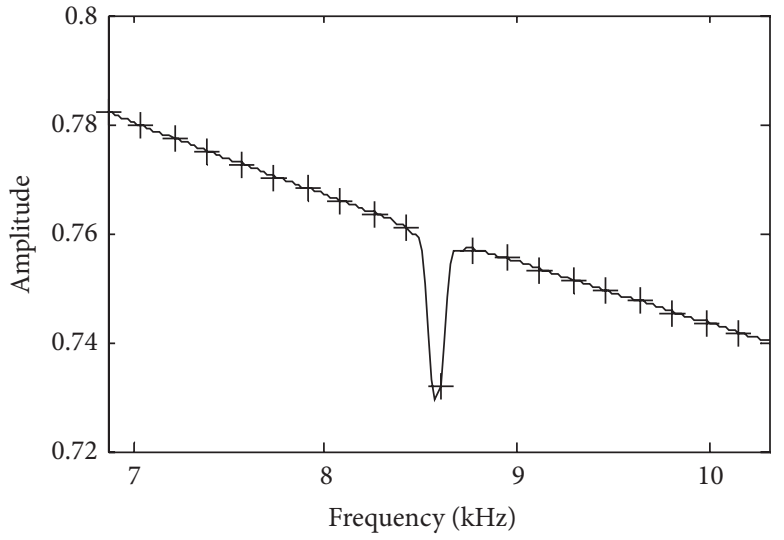

(b)

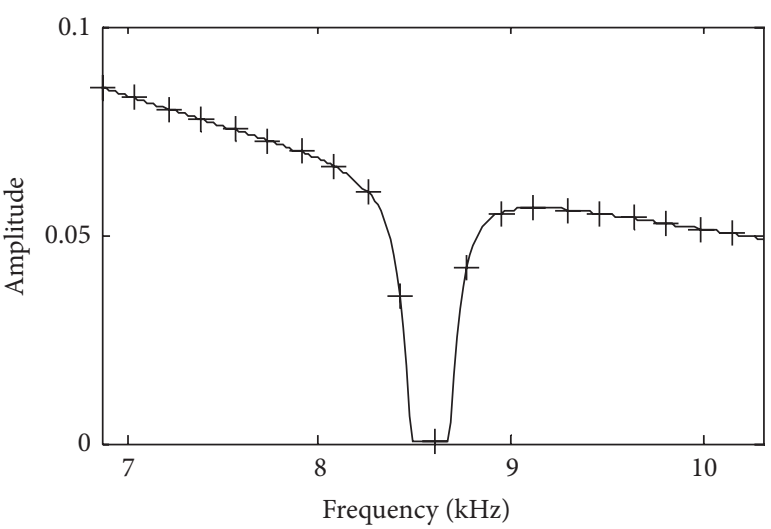

(d)

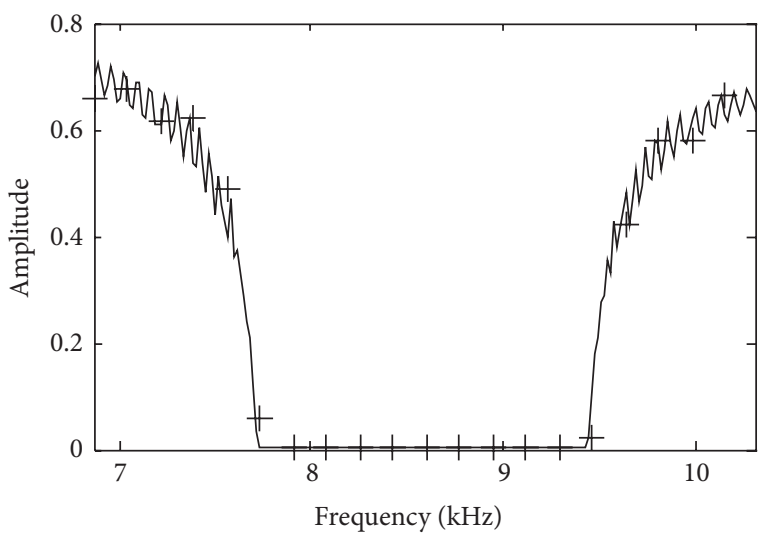

(f)

Figure 2: Reflection ((a), (c), (e)) and transmission ((b), (d), (f)) spectral responses, in magnitude, of a sinusoidally perturbed duct computed with (20) (solid line) and numerical results (crosses). ((a)-(b)) Case (i); ((c)-(d)) Case (ii); ((e)-(f)) Case (iii).

absorption phenomenon a posteriori, typically due to the duct walls, by redefining the acoustic wavenumber

$$
\beta=\frac{\omega}{v}+i \alpha,
$$

where $\alpha(\omega)$ is the absorption coefficient computed with mean cross-section parameters and $v(\omega)$ is the corresponding phase velocity.

\section{Examples}

Some examples of the acoustic behavior for a sinusoidally perturbed duct, filled with air at standard conditions $\left(20^{\circ} \mathrm{C}\right.$, $1 \mathrm{~atm})$, are presented here. They reveal the link between the passbands/stopbands response and the waveguide geometrical parameters, and they permit inferring the validity limit of the approximate formula. 
Wave attenuation and dispersion are included too, using (22), and $\alpha(\omega)$ and $v(\omega)$ are computed according to the wide-tube approximation (well described by Tijdeman [22]). Closed-form solutions are compared with full waveform numerical methods.

We consider a sinusoidal duct with length $L$, parameterized by the radius of the circular cross-section $r$ :

$$
r(x)=s \cos (\gamma x)+s_{0}, \quad 0<x<L .
$$

The average radius $s_{0}$ is set to $2 \mathrm{~cm}$, and the radius perturbation frequency $\gamma / 2 \pi$ is set to $50 \mathrm{~m}^{-1}$.

The other parameters are set to the following values:

$$
\begin{aligned}
& \text { Case (i): } L=2 \mathrm{~m}, s=0.02 \mathrm{~mm} ; \\
& \text { Case (ii): } L=20 \mathrm{~m}, s=0.2 \mathrm{~mm} \text {; } \\
& \text { Case (iii): } L=2 \mathrm{~m}, s=2 \mathrm{~mm} .
\end{aligned}
$$

Figure 2 shows the reflection and transmission responses, in magnitude, computed with (20), for the different cases, in a frequency interval centered at the frequency $f_{\max }$. The wavelength corresponding to $f_{\max }$ is not much higher than the duct transverse dimension, as required.

The crosses in Figure 2 are computed with an "exact" onedimensional simulator, based on the computation method described by Munday et al. [15], but with transmission coefficients included: the agreement between the approximate and the exact solution is very good.

The parameters values have been chosen to progressively increase the $s / s_{0}$ ratio, since a hypothesis for the approximation is $s \ll s_{0}$ : the effect of increasing this ratio is to widen the stopband. Moreover, Case (ii) investigates the effect of extending the duct length $L$, which is the other geometric parameter of the periodic structure: a higher length results in a sharper transition of the stopband.

The difference between approximate and theoretical values is barely noticeable even when $s / s_{0}$ ratio is as large as $1 / 10$, which may be considered the limit of validity of this approximation. Furthermore, the analytic formula is able to correctly simulate the attenuation phenomenon (this is apparent in the transmission response) which was not theoretically justified, but simply added a posteriori.

The phases are not shown here, but they have been verified as well.

\section{Conclusion}

The acoustic spectral response of a sinusoidally perturbed hard-wall duct has been derived and given in a simple formula, by following the optical analog of Bragg gratings. The formula is the same as in optics, with the electromagnetic parameters replaced by their equivalent acoustic parameters and periodic duct geometry.

Results are valid for small cross-section perturbations and in this case successful comparisons with a numerical method are shown, even in case of wave attenuation.

The availability of simple analytical formulas permits a direct analysis of the link between the acoustic response and the duct geometrical parameters and the design of efficient modeling/inversion procedures in the fields of bore/pipe reconstruction, noise control, and so forth.

Finally it can be noticed that, even if the derivation strictly requires a sinusoidal perturbation, any cross-section deformation can be decomposed in sinusoidal functions and therefore the results can be applied, provided that the underlying hypotheses are satisfied, to a much broader range of scenarios.

\section{Acknowledgment}

The authors wish to thank professor Andrea Melloni for the profitable discussions on the Bragg gratings theory and for the comments and suggestions on the paper production.

\section{References}

[1] C. Elachi, "Waves in active and passive periodic structures: a review," Proceedings of the IEEE, vol. 64, no. 12, pp. 1666-1698, 1976.

[2] R. F. Salant, "Acoustic propagation in waveguides with sinusoidal walls," Journal of the Acoustical Society of America, vol. 53, no. 2, pp. 504-507, 1973.

[3] A. H. Nayfeh, "Sound waves in two dimensional ducts with sinusoidal walls," Journal of the Acoustical Society of America, vol. 56, no. 3, pp. 768-770, 1974.

[4] O. R. Asfar and A. H. Nayfeh, "Circular Waveguide with Sinusoidally Perturbed Walls," IEEE Transactions on Microwave Theory and Techniques, vol. 23, no. 9, pp. 728-734, 1975.

[5] J. T. Fokkema, "Reflection and transmission of elastic waves by the spatially periodic interface between two solids (theory of integral-equation method)," Wave Motion, vol. 2, no. 4, pp. 375393, 1980.

[6] O. R. Asfar and A. H. Nayfeh, "Stopbands of the first-order Bragg interaction in a parallel-plate waveguide having multiperiodic wall corrugations," IEEE Transactions on Microwave Theory and Techniques, vol. 28, no. 11, pp. 1187-1191, 1980.

[7] A. El-Bahrawy, "Stopbands and passbands for symmetric rayleigh-lamb modes in a plate with corrugated surfaces," Journal of Sound and Vibration, vol. 170, no. 2, pp. 145-160, 1994.

[8] S. Banerjee and T. Kundu, "Elastic wave propagation in symmetrically periodic sinusoidal waveguide," in Health Monitoring and Smart Nondestructive Evaluation of Structural and Biological Systems III, vol. 5394 of Proceedings of SPIE, pp. 89-98, March 2004.

[9] S. Banerjee and T. Kundu, "Symmetric and anti-symmetric Rayleigh-Lamb modes in sinusoidally corrugated waveguides: an analytical approach," International Journal of Solids and Structures, vol. 43, no. 21, pp. 6551-6567, 2006.

[10] A. H. Nayfeh and O. R. Asfar, "Parallel-plate waveguide with sinusoidally perturbed boundaries," Journal of Applied Physics, vol. 45, no. 11, pp. 4797-4800, 1974.

[11] A. H. Nayfeh and O. A. Kandil, "Propagation of waves in cylindrical hard-walled ducts with generally weak undulations," AIAA Journal, vol. 16, no. 10, pp. 1041-1045, 1978.

[12] P. Latinopoulos and D. Tolikas, "Propagation of acoustic waves in ducts with varying cross sections and viscous mean flow," Computer Methods in Applied Mechanics and Engineering, vol. 23, no. 2, pp. 215-224, 1980. 
[13] A. Boström, "Acoustic waves in a cylindrical duct with periodically varying cross section," Wave Motion, vol. 5, no. 1, pp. 59-67, 1983.

[14] P. D. C. King and T. J. Cox, "Acoustic band gaps in periodically and quasiperiodically modulated waveguides," Journal of Applied Physics, vol. 102, no. 1, Article ID 014902, 2007.

[15] J. N. Munday, C. B. Bennett, and W. M. Robertson, "Band gaps and defect modes in periodically structured waveguides," Journal of the Acoustical Society of America, vol. 112, no. 4, pp. 1353-1358, 2002.

[16] B. N. Nagarkar and R. D. Finch, "Sinusoidal horns," Journal of the Acoustical Society of America, vol. 50, no. 1, pp. 23-31, 1971.

[17] D. J. Griffiths and C. A. Steinke, "Waves in locally periodic media," American Journal of Physics, vol. 69, no. 2, pp. 137-154, 2001.

[18] F. J. P. Lau and L. M. B. C. Campos, "On the effect of wall undulations on the acoustics of ducts with flow," Journal of Sound and Vibration, vol. 270, no. 1-2, pp. 361-378, 2004.

[19] M. A. Hawwa, "Acoustic wave blocking in a duct with a chirped periodic wall," Arabian Journal for Science and Engineering, vol. 29, no. 1C, pp. 113-124, 2004.

[20] H. Kogelnik, "Theory of optical waveguides," in Guided-Wave Optoelectronics, T. Tamir, Ed., pp. 60-65, Springer, 1988.

[21] T. Erdogan, "Fiber grating spectra," Journal of Lightwave Technology, vol. 15, no. 8, pp. 1277-1294, 1997.

[22] H. Tijdeman, "On the propagation of sound waves in cylindrical tubes," Journal of Sound and Vibration, vol. 39, no. 1, pp. 1-33, 1975. 


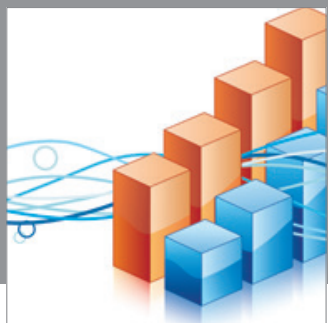

Advances in

Operations Research

mansans

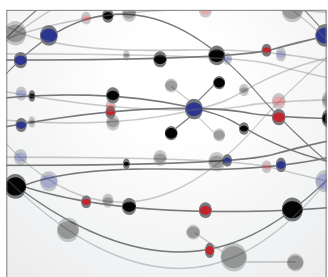

The Scientific World Journal
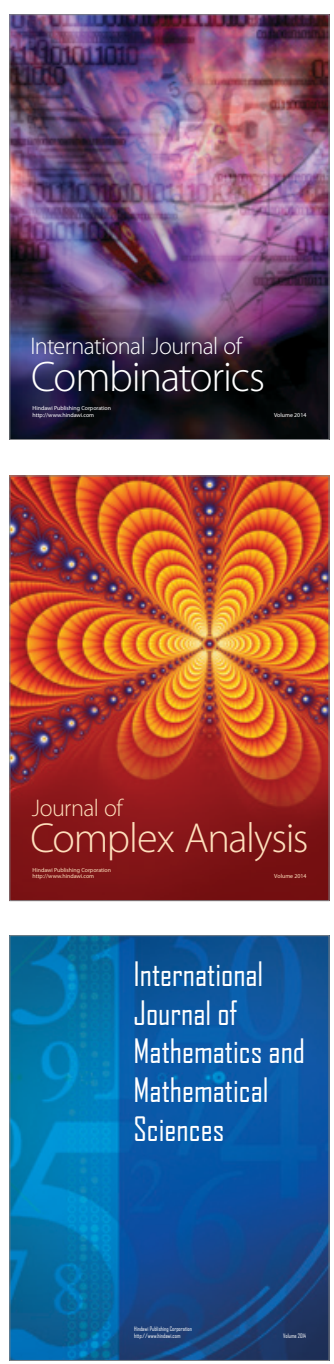
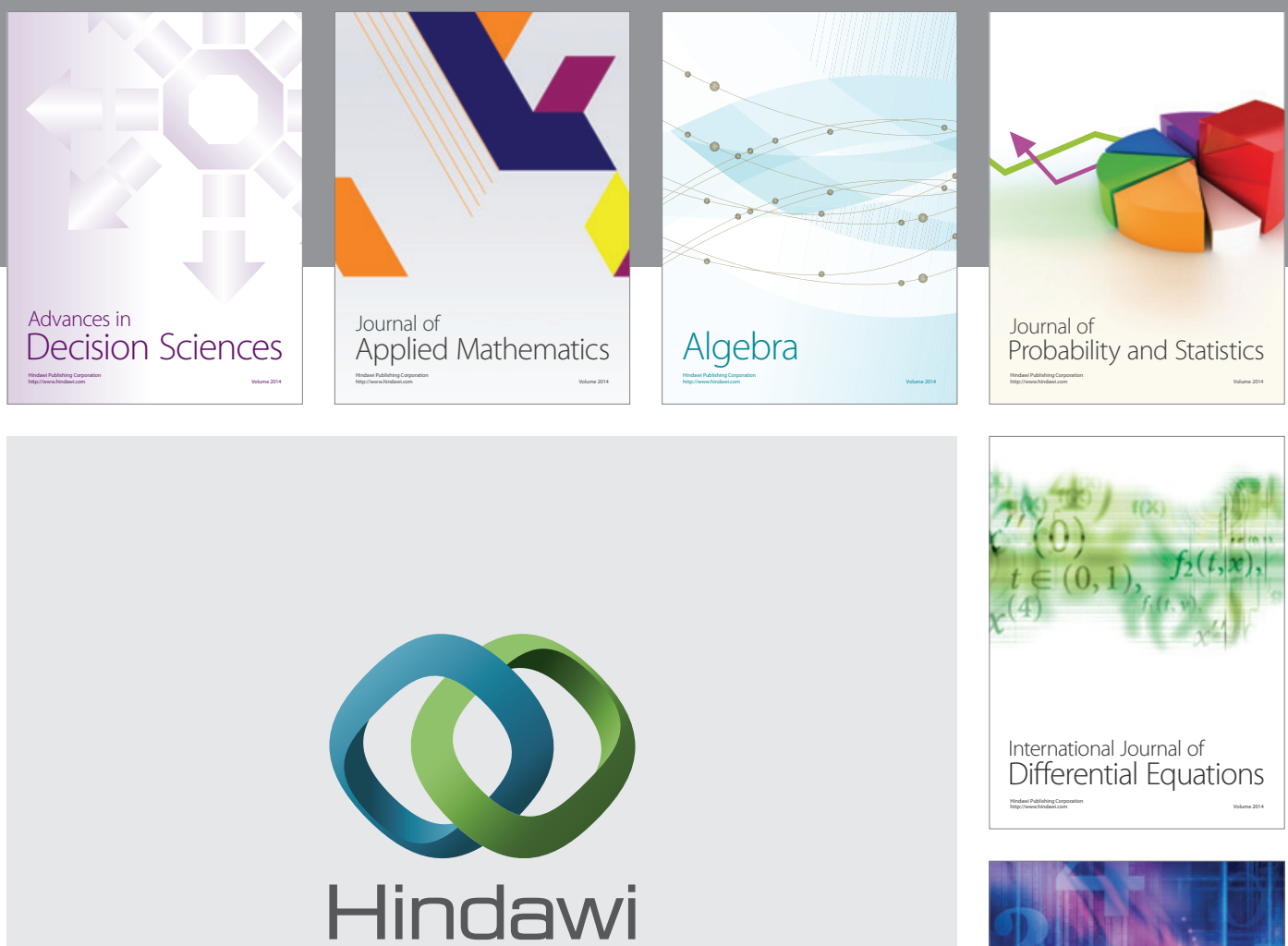

Submit your manuscripts at http://www.hindawi.com
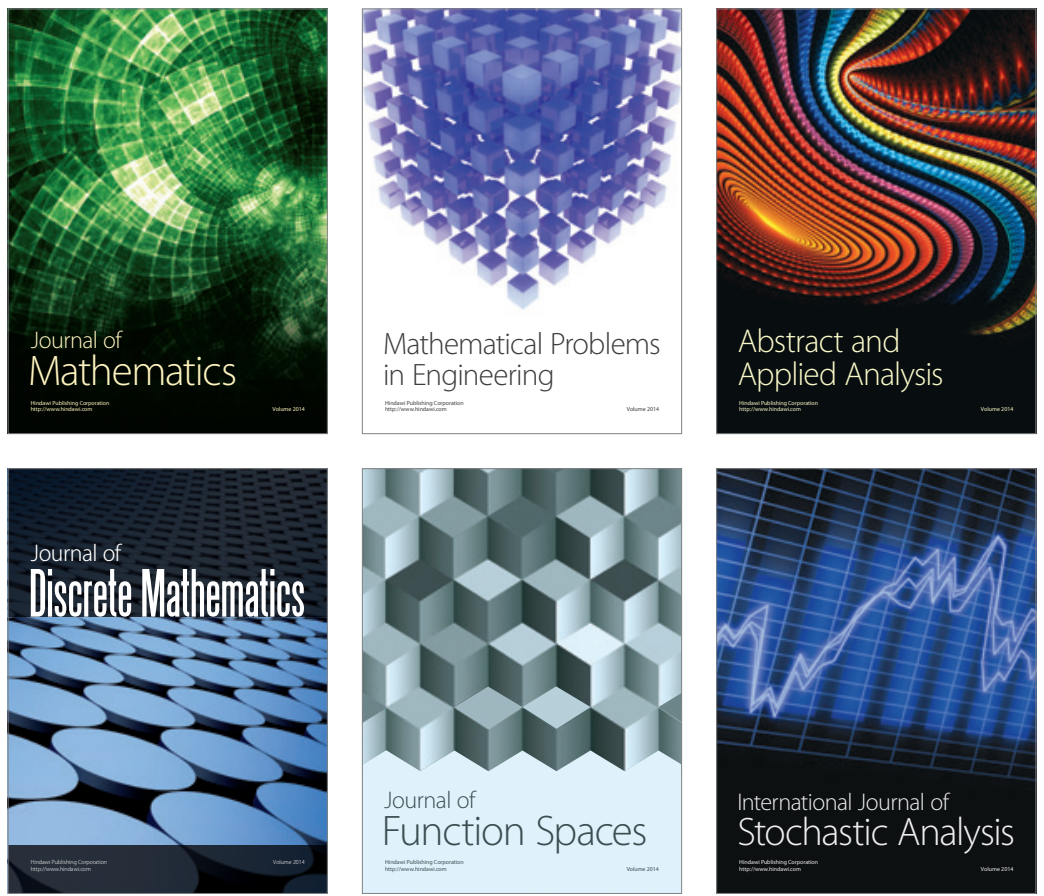

Journal of

Function Spaces

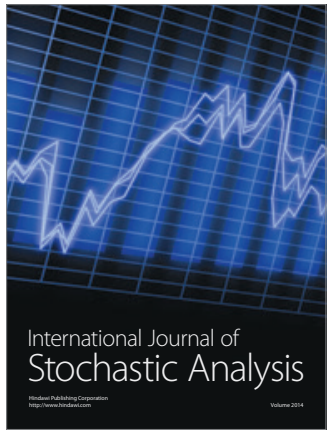

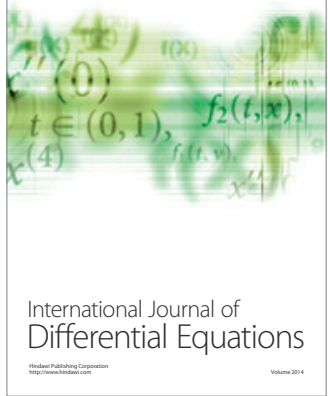
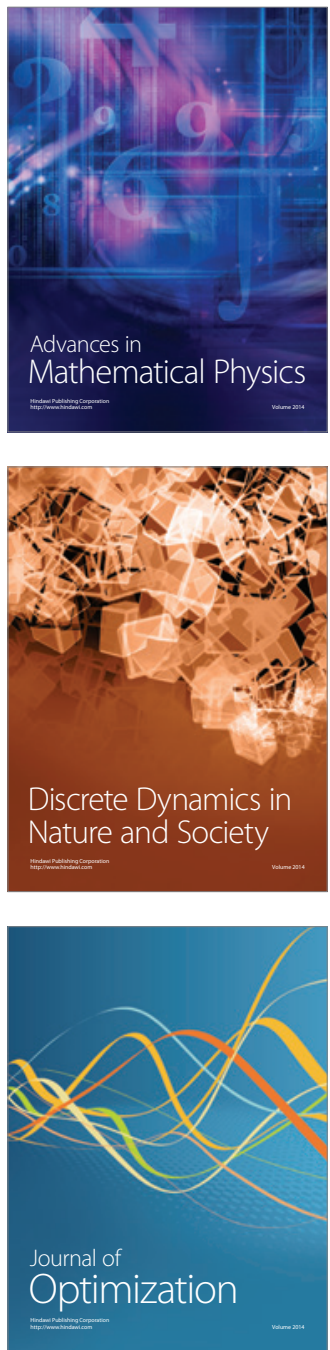